\title{
Design of intelligent high-voltage power supply for many kinds of
}

\section{radiation detector}

\author{
Luning Chen ${ }^{1, a *}$, Zheng Gao ${ }^{2, b}$, Yishan Jiang ${ }^{3, c}$, Weijie Xie ${ }^{4, d}$, Feng Xiao ${ }^{5, e}$ \\ 1,2,3,4,5 Navy Submarine Academy, China \\ acln15@mails.tsinghua.edu.cn, ${ }^{b}$ gaozheng@pku.org.cn, ${ }^{c j y s 130 @ 163 . c o m ~}$ \\ dWJxie64@hotmail.com, ${ }^{e} 350988058 @ q q . c o m$
}

Key words: Radiation detector, High -Voltage Power, LPC1768

\begin{abstract}
Different radiation detectors need different high-voltage values, and in this paper, the design of intelligent high-voltage power supply for many kinds of radiation detector is introduced, based on single-chip LPC1768, both the software and hardware circuit are adopted to achieve those functions of the power supply. Tests show that this power supply can completely be used in the portable radiation detection equipment that can link many kinds of nuclear radiation detectors. This power supply has many advantages in the using result, such as highly stability and intelligence, light weight and little volume, the reducing production cost etc.
\end{abstract}

\section{Introduction}

These nuclear radiation detectors, such as ionization chamber, proportional counter and GM counters and Scintillation detector etc, are commonly used in nuclear instrumentation, which must adopt high-voltage power supply system in the application process, and according to the different types of radiation detection, the value of operating voltage is also different. The accuracy of work voltage becomes the direct proportion with the efficiency and the stability of detector. In this paper, the design of intelligent high-voltage power supply for many kinds of radiation detector is introduced: based on single-chip LPC1768, both the software and hardware circuit are adopted to achieve those functions of the power supply. The output of the high-voltage power is accurate and reliable, and the volume is small. This power supply can completely be used in the portable radiation detection equipment that can link many kinds of nuclear radiation detectors.

\section{The system forms and working principle}

The intelligent high-voltage power supply includes SCM LPC1768, high-voltage module, high-voltage control circuit, high-voltage measuring and display driving circuit. The high-voltage module is used to export wide scope high-voltage value, which ranges from - $1000 \mathrm{~V}$ to $+1400 \mathrm{~V}$, and can supply many kinds of radiation detectors, such as ionization chambers, proportional counters, GM counters and Scintillation detector. High-voltage control circuit can adjust the output of high-voltage module by software, and so realize switching high-voltage when the detector exchanges. The different detector can obtain proper high-voltage for itself. High-voltage measuring and display driving circuit can real-time measure and show the high-voltage value on the LCD screen. The principle block diagram of the intelligent high-voltage power supply is shown in Fig 1. 


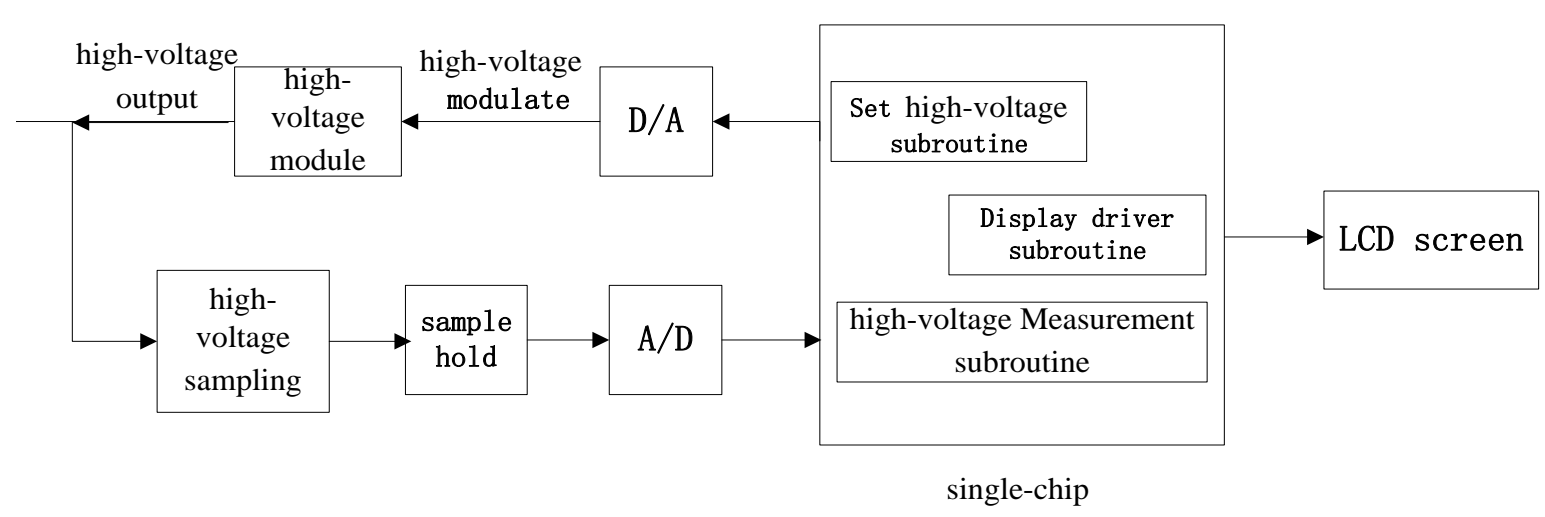

Fig. 1 The principle block diagram of the intelligent high-voltage power supply

\section{The design of hardware}

\section{SCM LPC1768}

SCM LPC1768 is based on ARM Cortex-M3 microcontrollers of NXP Company. The LPC1768 is applied to processing requirement of high integration and low power consumption. The LPC1768 operate at CPU frequencies of up to $100 \mathrm{MHz}$. The ARM Cortex-M3 CPU incorporates a 3-stage pipeline and uses Harvard architecture with separate local instruction and date buses as well as a third bus for peripherals. The peripheral complement of the LPC1768 includes up to $512 \mathrm{kB}$ of flash memory, up to $64 \mathrm{~kb}$ of data memory, Ethernet MAC, 4UARTs, 2 SSP controllers and 2 SPI interface,8-channel 12-bit ADC,10-bit DAC, and up to 70 general purpose I/O pins. [1]

\section{High-voltage module}

The high-voltage power supply for radiation detectors, whose electric current value is generally small from hundreds of microampere to a few milliamps, belongs to a kind of low power supply. In order to reduce the volume and weight, achieving high-voltage is generally not directly to boost and rectify $220 \mathrm{~V} 50 \mathrm{~Hz}$ voltage. The process of obtaining high-voltage is that the stable low-voltage via DC - AC conversion becomes a high-frequency AC high-voltage, which is then by voltage double rectifier filter to become a DC high-voltage. [2] In order to get the high-voltage, which wide ranges from $-1000 \mathrm{~V}$ to $+1400 \mathrm{~V}$, it must be used a positive high-voltage power whose output ranges from $0 \mathrm{~V}$ to $+1400 \mathrm{~V}$ and a negative high-voltage power whose output ranges from $-1000 \mathrm{~V}$ to $0 \mathrm{~V}$. The positive high-voltage power and the negative high-voltage power are shown in Fig.2. The stability of the two high-voltage powers must achieve $0.05 \sim 0.01 \% / \mathrm{h}$. The output resistance is less than $100 \Omega$, and the temperature coefficient is less than $20 \sim 100 \mathrm{ppm} /{ }^{\circ} \mathrm{C}$.

The output value is determined by the "adjustment" pin of the high-voltage module. The "adjustment" pin connects with one of the output end of analog voltage of high-voltage control circuit. While the output of analog voltage is $0 \mathrm{~V}$, the output of high-voltage module is the lower limit of range. And while the output of analog voltage is full range, the output of high-voltage module is the upper limit of range. To control the output value of the high-voltage module, simply set a voltage based on scale to the "adjustment" pin. Set a digital value in the microcontroller program, that be converted into analog voltage by high-voltage control circuit and then inputted to "adjustment" pin of high-voltage module .By this way, a proper voltage can be achieved from the output pin of high-voltage module. 


\section{High-voltage control circuit}

SPI serial bus of LPC1768 sends high-voltage control instructions, converted into a high-voltage control signal to regulate positive and negative high-voltage power module. [3] The main form of high-voltage control circuit is an AD5724 chip. High-voltage control circuit is shown in Fig.2.

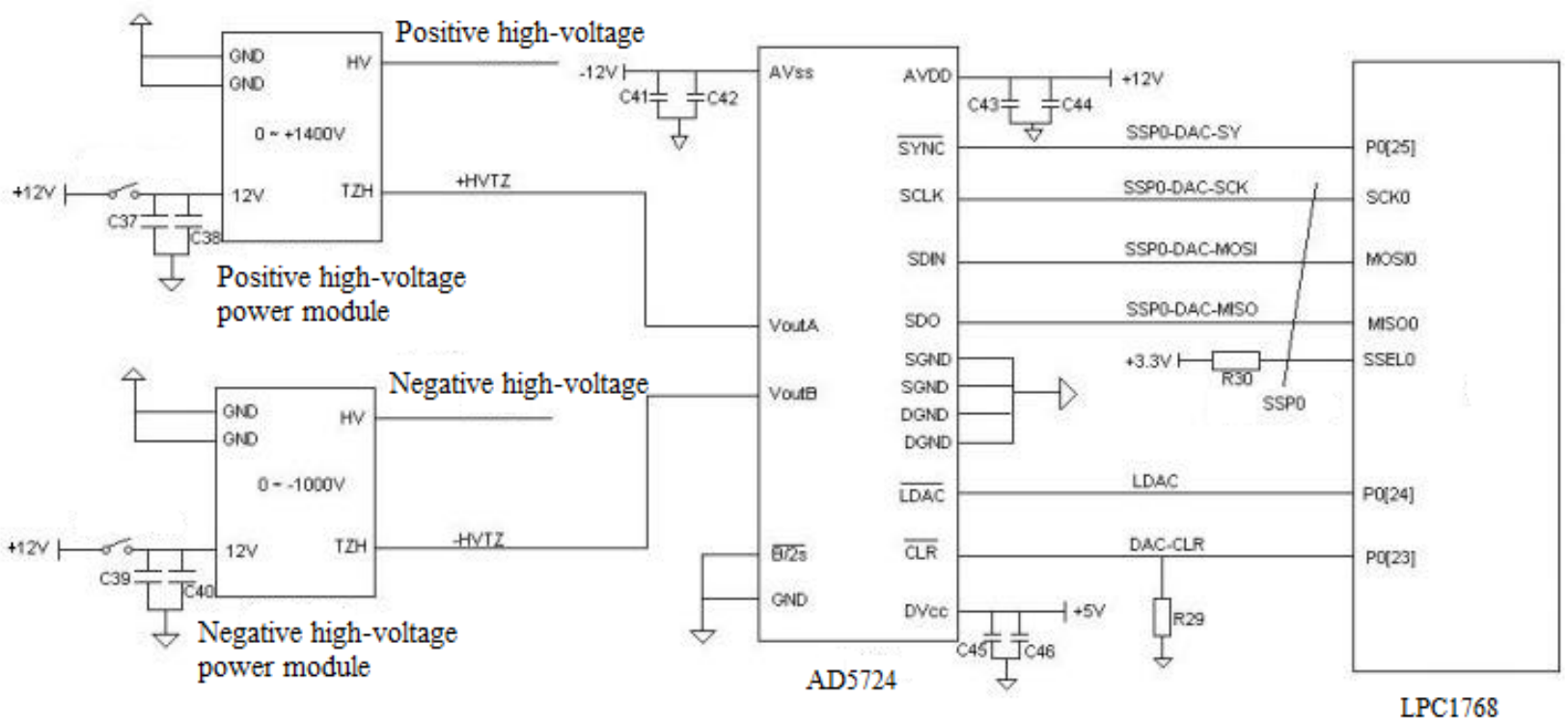

Fig.2 High-voltage control circuit

AD5724 is quad, 12-bit, serial input, voltage output digital-to-analog converter. In this paper, it operates dual supply voltages $\pm 12 \mathrm{~V}$. Its external reference power is $2.5 \mathrm{~V}$, and nominal full-scale output range can be selected by software. SCM LPC1768 adopts SSP0 interface, and is set as the main engine. The pins of SSP0 interface of LPC1768 are connected with corresponding pins of AD5724. According to the description of AD5724 chip in the manual, in order to make AD5724 normally operate, we must accurately set SYNC, LDAC and CLR. These three pins should be respectively linked with P0 [25], P0 [24] and P0 [23] of LPC1768. Configure P0 [25], P0 [24] and $\mathrm{P0}$ [23] as general I/O pins during the initialization of SCM program. And then set different voltage value that associated with the corresponding detector in main program. The choice of detector's type is controlled by the key of external interrupt. By this way, high-voltage control circuit can control the output value of high-voltage module. [4]

\section{High-voltage measuring circuit}

High-voltage measuring circuit is shown in Fig.3. First, the high-voltage value generated by high-voltage module pass a voltage divider circuit. (In Fig.3, R5, R6 and RP1 compose a voltage divider circuit. R7, R8 and RP2 compose a voltage divider circuit. RP1 and RP2 is potentiometer that can fine adjust the high-voltage sample. The value of these large resistances, R5 and R7, is $33 \mathrm{M} \Omega$.)And then, these high-voltage samples respectively obtained from two voltage divider circuits pass sample hold circuits (R13 and OP3A compose a sample hold circuit, R16 and OP2A compose a sample hold circuit).The signals of output voltage are inputted to analog input channels of the AD7327 chip, and then by the way of SPI serial bus data mode, into the LPC1768 microcontroller in there to be processed by software. Last, the high-voltage measurements can be directly displayed on the LCD screen. 


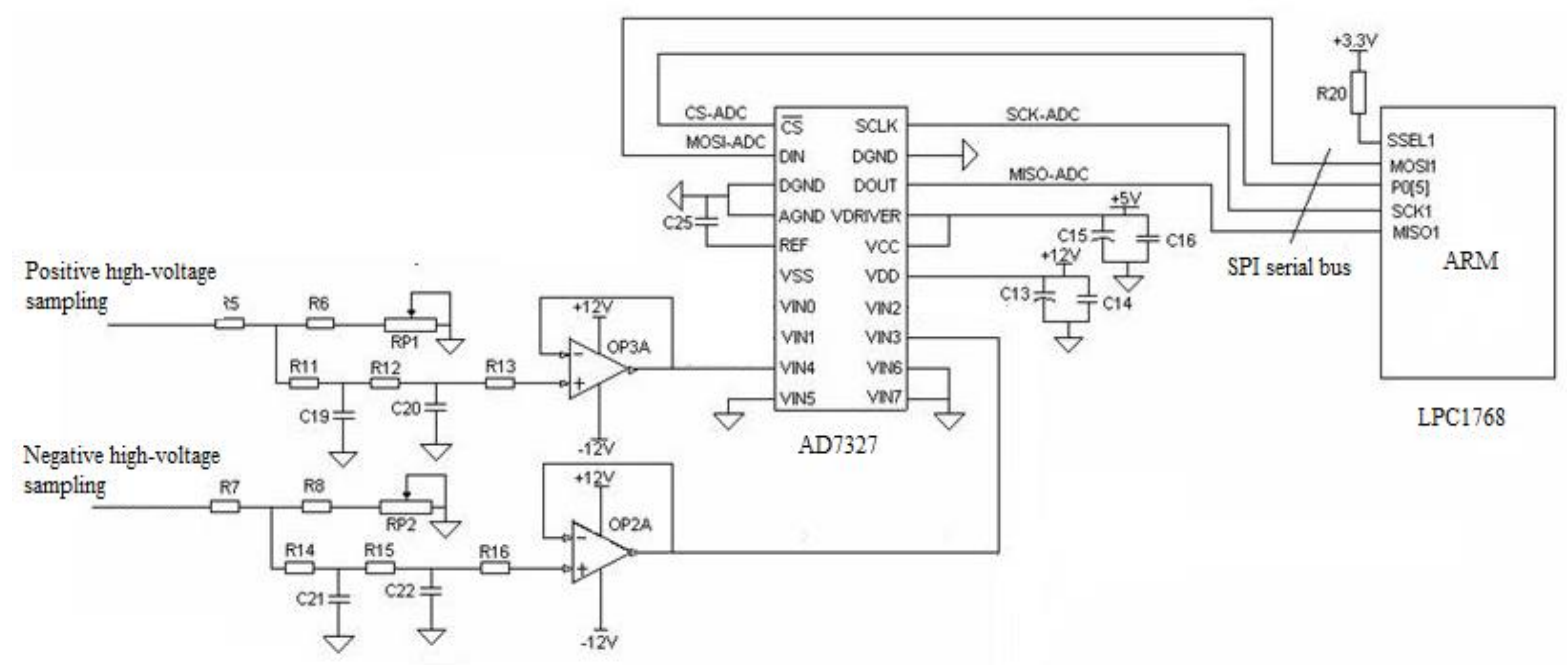

Fig. 3 High-voltage measuring circuit

\section{Software design}

The software procedure of high-voltage power of radiation detector is in order to provide the different working high voltage to different kinds of radiation detectors.

Program pseudo-code is as follows:

1 system initialization

2 initialize DAC

a. Configure P0.17 for SSP0-DAC-MISO

b. Configure P0.18 for SSP0-DAC-MOSI

c. Configure P0.25 for DAC-SY

d. Configure P0.23, P0.24 for universal pin

e. Configure P0.23, P0.24 for the output pin

3 initialize IO

4 LCD screen initialization

5.while 1

a.do gc_SETIndex $\leftarrow$ channel number

b. if gc_SETIndex = 1 then call SETDAC0 () // set the high-voltage adjusting value for channel1 else if gc_SETIndex $=2$ then call SETDAC1 () // set the high-voltage adjusting value for channel2

else if $\mathrm{gc}$ _SETIndex $=\mathrm{n}$ then call SETDAC7 () // set the high-voltage adjusting value for channel $n$

c. if time is greater than $1 \mathrm{~s}$

1 timer flag is cleared

timer re-timing

2. then call MesureAnalog () // call measuring function of high-voltage value

3. call Displayfunction (gc_displayindex) // call display function

\section{Analyses of test results}

To prove that the system is accurate and reliable, it was tested with a digital multimeter. [5] The test object is negative high-voltage and positive high-voltage, such as $-800 \mathrm{~V},-400 \mathrm{~V}, 420 \mathrm{~V}, 450 \mathrm{~V}$ 
and $1250 \mathrm{~V}$. The test results were shown in table I. V1 is the value of output of high-voltage module, which was measured with the digital multimeter. V2 is the value shown in LCD of high-voltage measuring circuit.

Table I The compare of output of high-voltage module and the value shown in LCD

\begin{tabular}{ccccc|cccccc}
\hline & \multicolumn{6}{c|}{ negative high-voltage $/ \mathrm{V}$} & \multicolumn{5}{c}{ positive high-voltage/V } \\
\cline { 2 - 10 } & $\mathrm{V}_{1}$ & $\mathrm{~V}_{2}$ & $\mathrm{~V}_{1}$ & $\mathrm{~V}_{2}$ & $\mathrm{~V}_{1}$ & $\mathrm{~V}_{2}$ & $\mathrm{~V}_{1}$ & $\mathrm{~V}_{2}$ & $\mathrm{~V}_{1}$ & $\mathrm{~V}_{2}$ \\
\hline 1 & -801.5 & -801.45 & -400.5 & -402.35 & 422.2 & 422.35 & 451.3 & 452.20 & 1252.4 & 1253.32 \\
2 & -800.5 & -800.25 & -399.3 & -400.28 & 420.2 & 420.25 & 450.2 & 450.38 & 1251.5 & 1252.46 \\
3 & -800.4 & -800.43 & -401.3 & -400.20 & 421.3 & 422.39 & 452.2 & 452.32 & 1252.4 & 1253.25 \\
4 & -799.4 & -800.28 & -400.3 & -401.20 & 419.2 & 420.18 & 451.2 & 450.18 & 1249.5 & 1250.35 \\
5 & -800.8 & -801.12 & -400.3 & -401.16 & 420.2 & 421.05 & 449.3 & 450.38 & 1250.5 & 1251.28 \\
\hline
\end{tabular}

Seeing from table1, the maximum error value of output of high-voltage module is $0.25 \%$, and the maximum error value of high-voltage measuring circuit is $0.27 \%$.According to the test results, it can been concluded that the intelligent high-voltage power supply is accurate and reliable.

\section{Conclusions}

In the article, single-chip and various peripheral chips are used in the design of the intelligent high-voltage power supply, through hardware and software controlling output of high-voltage modules. It has been to the needs of various types of high-voltage detector. At present, the power supply has been successfully applied to intelligent nuclear detector instruments. Practice has proved that: The power supply system improves the stability and intelligence of nuclear detection equipment, while small size and light weight. So the power supply system has broad applied prospects and huge economic value.

\section{Reference}

[1] MA Zhong-mei, XU Yan, YE Qing-lin. ARM Cortex microprocessor tutorial[M].Beijing: Beihang University Press

[2] WANG Jing-jin, FAN Tian-min, QIAN Yong-kang. Nuclear electronics[M].Beijing: The technique of Atomic Energy Press. 1984

[3] TANG Yun-bao, LI Yin-ling. Time Scale Sampling System Based on LPC1768 and AD7656. Microcontrollers \& Embeded[J].2010(8):37-40

[4] RUAN Lin-bo, CAO Jin-yun, HEI Dong-wei. Design of multiple-channel high voltage DC power device based on cornputer control[J]. High Voltage Engineering, 2003,29(2):19-20

[5] CHEN Yuan-yuan, WANG Lei, CAI Ting, CHENG Yi. Design of Multi-channel Power Monitor for Nuclear Instruments[J]. Nuclear Electronics \& Detection Technology.2015,35 (2):193-197 